\title{
Chaotically Spiking Canards in an Excitable System with 2D Inertial Fast Manifolds
}

\author{
Francesco Marino, ${ }^{1}$ Francesco Marin, ${ }^{1}$ Salvador Balle, ${ }^{2}$ and Oreste Piro ${ }^{2,3}$ \\ ${ }^{1}$ Dipartimento di Fisica, Università di Firenze, INFN, Sezione di Firenze, and LENS, \\ Via Sansone 1, I-50019 Sesto Fiorentino (FI), Italy \\ ${ }^{2}$ Institut Mediterrani d'Estudis Avançats, CSIC-UIB, E-07071 Palma de Mallorca, Spain \\ ${ }^{3}$ Center for Studies in Physics and Biology, Rockefeller University, New York, New York 10021, USA
}

(Received 6 November 2006; published 13 February 2007)

\begin{abstract}
We introduce a new class of excitable systems with two-dimensional fast dynamics that includes inertia. A novel transition from excitability to relaxation oscillations is discovered where the usual Hopf bifurcation is followed by a cascade of period doubled and chaotic small excitable attractors and, as they grow, by a new type of canard explosion where a small chaotic background erratically but deterministically triggers excitable spikes. This scenario is also found in a model for a nonlinear Fabry-Perot cavity with one pendular mirror.
\end{abstract}

DOI: 10.1103/PhysRevLett.98.074104

PACS numbers: 05.45. $-\mathrm{a}, 42.65 .-\mathrm{k}, 42.65 . \mathrm{Sf}$

Excitability is an important property of a variety of systems studied in many fields, including neural-sciences, chemistry, several branches of physics, and engineering, etc. Neural cells [1], cardiac tissues [2], autocatalytic reactions [3], semiconductor microcavities [4], among a much larger variety of examples, exhibit excitable dynamics characterized by the existence of one stable steady state that can be forced to spike by external stimuli of amplitude above a given relatively small threshold. The excitable spikes have well defined shape, amplitude, and duration that do not depend on the detailed nature of the stimulus and are followed by a characteristic refractory time interval during which the system is not responsive to any new, above-threshold stimuli.

In all the cited examples, excitability is the result of multiple time scale dynamics [5]. The van der PolFitzHugh-Nagumo (vdPFN) system [6], originally conceived as a simple description of neural activity, paradigmatically illustrates this fact:

$$
\begin{aligned}
& \dot{x}=y+x-\frac{x^{3}}{3}, \\
& \dot{y}=-\varepsilon(x-a) .
\end{aligned}
$$

When $\varepsilon \ll 1$, the typical rates of variation for $x$ and $y$ are very different. Notice, however, that $x$ can vary at the same slow rate as $y$ in an $\varepsilon$-wide band of phase space around the nullcline of $x$, the curve $y=\left(x^{3} / 3-x\right)$ also referred to as the slow manifold of the motion. Thus, the vdPFN dynamics can be decomposed into a sequence of periods of slow motion taking place near the slow manifold separated by fast relaxations along the $x$ direction described by Eq. (1) with $y=$ const. The last constraint defines a onedimensional fast manifold [7].

The only steady state $(x, y)=\left(a, a^{3} / 3-a\right)$ of the vdPFN model is globally stable for $|a|>1$ and unstable for $|a|<1$. When the fixed point is stable, the system is excitable as described above, while for $|a|<1$, it performs self-sustained relaxation oscillations. In Eqs. (1) and (2), the transition from excitability to relaxation oscillations takes place at $a=a_{c}= \pm 1$, initially through a supercritical Hopf bifurcation giving birth to a small quasiharmonic limit cycle of amplitude growing as $\sqrt{\left|a_{c}\right|-|a|}$ near $a_{c}$. However, the splitting of time scales limits the transitional regime to a narrow range of $a$ of order $\varepsilon$ below $\left|a_{c}\right|$. Out of this range, the frequency and the amplitude of the limit cycle abruptly change to those of the relaxation oscillations regime. These sudden changes are known as canard explosions [8], and their physical implications are currently under intense investigation [9-12].

In two-dimensional (2D) phase-spaces, this scenario of a single Hopf bifurcation followed by a canard explosion is the only possible. In contrast, higher-dimensional systems support more varied and complex dynamics with chaos, mixed-mode oscillations, erratic bursting, etc. Some 3D generalizations of the vdPFN model with a 2D slow manifold and 1D fast motions have appeared in electronics [13], laser physics [14], chemistry [15], ecology [16], etc. Surprisingly, however, excitable systems with onedimensional slow-manifolds and at least two-dimensional fast ones have not been studied so far.

This Letter aims to uncover surprising features in this class of systems. Inspired by the dynamics of a nonlinear Fabry-Perot cavity with one pendular mirror, we introduce a $3 \mathrm{D}$ extension of the vdPFN model where the fast motion includes inertial terms, therefore occurring on a twodimensional fast manifold. We then show that such inertia leads to a radically new scenario for the transition from excitability to relaxation oscillations. The initial Hopf bifurcation is now followed by a cascade of perioddoubling bifurcations producing a sequence of small periodic and chaotic attractors, that develops before relaxation oscillations arise. On each of these attractors, the system displays excitable behavior, but as the mean amplitude of the chaotic attractors grows, a canard regime sets in where the small chaotic background spontaneously triggers ex- 
citable spikes in an erratic but deterministic sequence. We finally demonstrate this scenario in a detailed physical model for pendular optical cavities where a competition between the radiation pressure on the mirrors and their thermal expansion caused by the partially absorbed intracavity power provides the basic ingredient for a vdPFN dynamics while the mechanical reaction of the pendular mirror introduces inertia in the fast motion. Since pendular cavities are important for applications in quantum optics experiments [17,18] and gravitational wave detection $[19,20]$, the interest of these results extends well beyond the domain of nonlinear dynamics.

As a paradigm to study the effects of inertia on excitable dynamics, let us introduce a minimal model by extending the vdPFN to 3D with an acceleration term in the equation for the fast-variable:

$$
\begin{gathered}
k \ddot{x}+\dot{x}=y+x-\frac{x^{3}}{3}, \\
\dot{y}=-\varepsilon(x-a) .
\end{gathered}
$$

As in the noninertial case, $a$ is the control parameter, and $\varepsilon \ll 1$ separates the time scales. The additional parameter $k$ determines the relative importance of the inertial motion as well as its time scale, taking us to the original vdPFN in the limit $k \rightarrow 0$. Here, however, we are interested in the range $1 \gtrsim 1 / k \gg \varepsilon$ where we shall see that fast relaxation and inertia are comparably important to the fast dynamics.

For this range of $k$, an inspection of Eqs. (3) and (4) reveals that, since $y$ typically changes at a much slower rate than $x$ and $\dot{x}$, the motion still splits into fast and slow epochs [7]. During the fast epochs, the change of $y$ can be neglected, and the dynamics be described by Eq. (3) with $y=$ const as a parameter. The "fixed points" of this dynamical subsystem lay on the one-dimensional $S$-shaped manifold $\Sigma=\left\{\left(x_{y}, \dot{x}=0, y\right)\right\}$ defined by the equation $x_{y}^{3} / 3-x_{y}=y$. It is on this manifold, then, where the slow dynamics described by Eq. (4) can now take place. With respect to the fast dynamics (3) defined on the planes $y=$ const transversal to the slow manifold, the fixed points along $\Sigma$ are saddles if $x_{y}^{2}<1$, stable nodes if $1<x_{y}^{2}<1+$ $1 / 4 k$, and stable foci if $x_{y}^{2}>1+1 / 4 k$. Therefore, the slow manifold $\Sigma$ is composed of two attracting branches $\Sigma_{1}=$ $\Sigma \cap\{x>1\}$ and $\Sigma_{2}=\Sigma \cap\{x<-1\}$ separated by a repelling branch, $\Sigma_{3}=\Sigma \cap\{-1<x<1\}$.

In the limit $\varepsilon \rightarrow 0$, we can qualitatively describe the dynamics of Eqs. (3) and (4) with pretty much the same singular analysis valid for the noninertial case (1) and (2) [21]. Since the branches $\Sigma_{1,2}$ rapidly attract all neighboring trajectories - while $\Sigma_{3}$ repels them-most of the time, the motion has to take place along these branches. There, Eq. (4) dictates that $y$ grows for $x<a$ and decreases for $x>a$. When this prescription forces the system to reach any of the $y$-turning points $x= \pm 1$ on $\Sigma$, the trajectory forcibly abandons the slow manifold turning on the fast dynamics that drives it to the opposite stable branch. As in the vdPFN model, the only fixed point of the system is $(x, \dot{x}, y)=\left(a, 0, a^{3} / 3-a\right)$ which, as $a$ varies, also becomes unstable through a supercritical Hopf bifurcation of frequency $\omega=\sqrt{\varepsilon}$. Here, however, this bifurcation occurs at $a= \pm \sqrt{1+\varepsilon} \bar{k}$ regaining the noninertial value in the limit $\varepsilon \rightarrow 0$ under consideration. Hence, the fixed point is stable if it lies on the branches $\Sigma_{1,2}$ and unstable on $\Sigma_{3}$. In the first situation, attained with $|a|>1$, the dynamics is excitable: the system is bound to asymptotically rest on the globally attracting fixed point, but once there, it can be forced to temporarily jump to the opposite stable branch by means, for example, of a shortly lasting temporary reset of $a$ to a value across the nearest turning point $x= \pm 1$. During its return path to rest-which involves reaching first the turning point of this branch, jumping then back to the branch of the fixed point, and finally converging to itthe system is refractory, i.e., insensitive to any further perturbation. Conversely, when $|a|<1$, the system performs relaxation oscillations through a cycle composed by segments of the stable branches ending at the $x= \pm 1$ $y$-turning points followed by rapid jumps to the opposite branch.

Although the features described above are quite similar to those in the original vdPFN, the dynamics of Eqs. (3) and (4) is radically different. First of all, recall that as fixed points of the 2D fast subsystem (3), $\Sigma_{1,2}$ consists of stable foci, except for two short segments near the $y$-turning points $x= \pm 1$ where they become stable nodes. Therefore, except for these small regions, the trajectories near-but not strictly on-these branches are shrinking helicoids. This is particularly true immediately after the jumps. This spiralling behavior contrasts with the monotonic decay that can uniquely be expected in the noninertial case.

Even more interestingly diverse in our inertial excitable system is the transitional canard regime from excitability to relaxation oscillations. Notice first that the Hopf bifurcation takes place while the fixed point is still on the attracting branches $\Sigma_{1,2}$. Since the whole system is $3 \mathrm{D}$, there is room for a complex scenario that includes cascades of period doubled and chaotic attractors of small amplitude in an $\varepsilon$ neighborhood of these branches. This is illustrated by Fig. 1 where a bifurcation diagram similar to that of the logistic map is computed from our system varying $a$ over a small interval contiguous to the initial Hopf bifurcation. In each one of these multiplicity of attractors, the system is excitable: small perturbations elicit small responses from it that rapidly return back to the attractor [Fig. 2(a)] while sufficiently strong stimuli provoke a long excursion, insensitive to the details of the perturbation, to the opposite stable branch before returning to the attractor [Fig. 2(b)]. Moreover, during such excursion, the system is refractory to further stimuli as in the fixed point based excitability. We can thus dub this novel generalized scenario as "chaotic excitability."

As $a$ approaches the $y$-turning point, the mean amplitude of the attractors grows as seen in Fig. 1, until the chaotic 


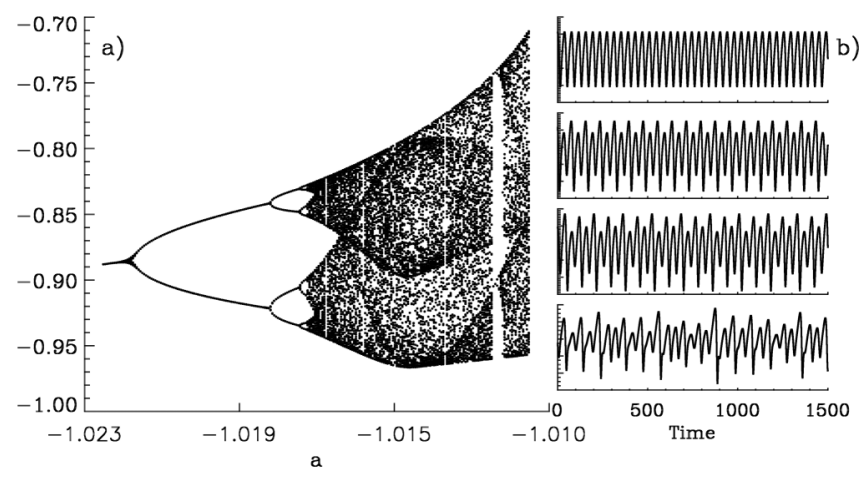

FIG. 1. (a) Bifurcation diagram for the peak values of $x$ as the parameter $a$ is varied in Eqs. (3) and (4). (b) Signal form of $x$ for $a=\{-1.0225,-1.019,-1.0172,-1.01\}$ from top to bottom. Everywhere, $k=3$ and $\varepsilon=0.03$.

fluctuations are sufficiently large to eventually trigger the fast dynamics. This results in an erratic-sensitive to initial conditions - sequence of canard spikes on top of a chaotic background (Fig. 3). Further varying $a$, the mean firing rate increases until a periodic regime is finally reached. Similar sequences have been experimentally observed in a ferroin-catalyzed Belousov-Zhabotinskii reaction performed in a continuous-stirred tank reactor [22]. This behavior is reminiscent of the one observed in the vdPFN model forced by random noise. There, the system responds by randomly spiking on a noisy background, with a mean firing rate that increases with the noise amplitude and with an exponentially vanishing interspike interval (ISI) distribution cutoff at the refractory time [23]. Here, in contrast, there are no external forces, the aperiodic background triggers excitable spikes in an erratic but completely deterministic sequence, and, on top of the exponential tail, the ISI histogram displays a complicated structure of sharp peaks (Fig. 3(b)) revealing the complex structure of unstable periodic orbits embedded in the chaotic attractor nonlinearly filtered by the excitability threshold.

The scenario described above is found in a detailed physical model of a high-finesse, pendular Fabry-Perot cavity in the presence of radiation pressure and photothermal effects. In such systems, both effects are significant and competitive: while the former increases the cavity length by pushing the mirrors, the latter decreases it [24] due to the thermal expansion of the mirrors. With an input
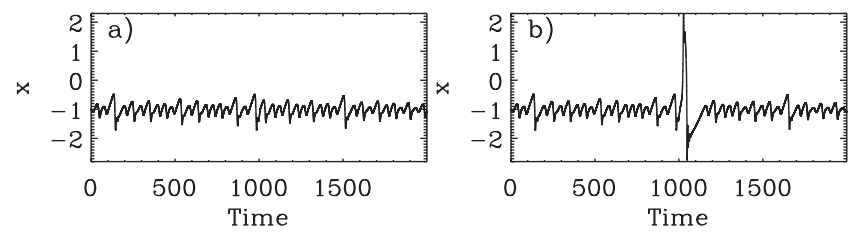

FIG. 2. System response to a $\delta$-like perturbation of amplitude $p_{0}$ delivered at $t_{0}=1000$ on the right hand side of Eq. (4) with $a=-1.01, k=3, \varepsilon=0.03$. (a) $p_{0}=8 \times 10^{-2}$. (b) $p_{0}=9 \times 10^{-2}$. field detuned to the red from the cavity resonance, the first effect tends to increase the intracavity optical power and the second to reduce it. Under appropriate conditions, the interplay between these effects operating at different time scales produces excitability and relaxation oscillations in the optical intensity [25]. Indeed, the physics involved is analogous to the one theoretically and experimentally observed in semiconductor microcavities where the intracavity optical path nonlinearly depends on the optical intensity via the refractive index [12]. However, while in semiconductors, the fast and slow motions are strongly overdamped, in pendular cavities, the mechanical reaction of the pendular mirror is important. The model thus requires an inertial term in the equation for the fast variable and reads [25]

$$
\begin{aligned}
\ddot{\phi}+\frac{1}{Q} \dot{\phi} & =-\phi+\frac{\alpha}{1+\left(\delta_{0}+\phi+\theta\right)^{2}} \equiv F(\phi, \theta), \\
\dot{\theta} & =-\tilde{\varepsilon}\left[\theta+\frac{\beta}{1+\left(\delta_{0}+\phi+\theta\right)^{2}}\right]
\end{aligned}
$$

where $\phi$ and $\theta$ are the detuning variation due to radiation pressure and photothermal effect, respectively, $Q$ is the mechanical quality factor of the pendulum, $\delta_{0}$ the coldcavity detuning, $\tilde{\varepsilon}$ the mirror temperature rate of change, and $\alpha$ and $\beta$ measure the strength of the radiation pressure and photothermal effects [26].

When $\tilde{\varepsilon} \ll 1$, the evolution of $\theta$ is slow with respect to that of the other variables. By separating fast and slow motion in the limit $\tilde{\varepsilon} \rightarrow 0$, one can find that the structure and the stability of the slow manifold is analogous to that of Eqs. (3) and (4) and that the slow motion is restricted on the curve $F(\phi, \theta)=0$ (S-shaped line in Fig. 4). The steady
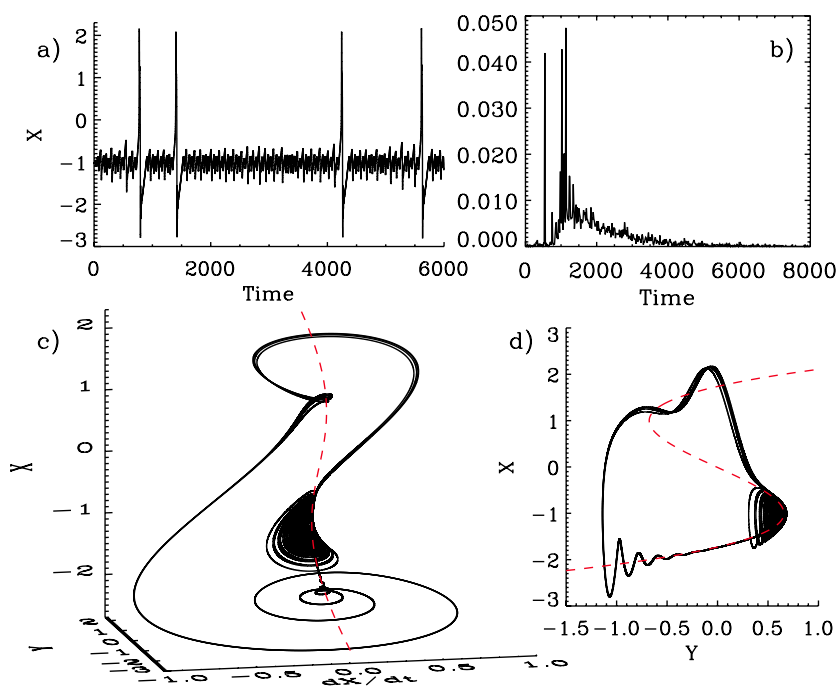

FIG. 3 (color online). (a) Temporal evolution of $x$ in the chaotically spiking regime. (b) The corresponding ISI distribution. (c) Phase-space trajectory with the slow manifold in dashed line style and (d) projection of c) on the $(x, y)$ plane. In all panels, $a=-1.00927, k=3, \varepsilon=0.03$. 


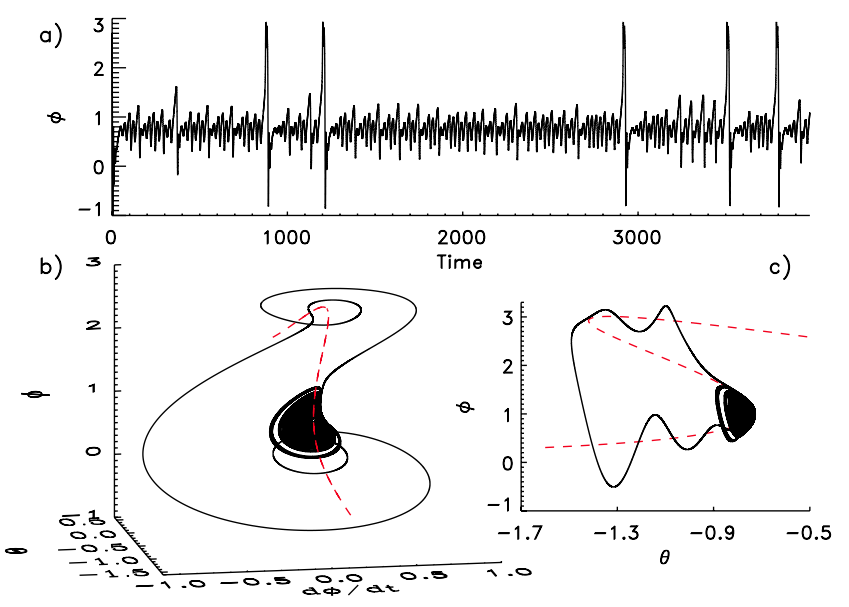

FIG. 4 (color online). (a) Temporal evolution of $\phi$ in the chaotically spiking regime. (b) The corresponding phase-space trajectory with the slow manifold in dashed line style and (c) its projection on the $(\theta, \phi)$ plane. In all panels, $\delta_{0}=-1.676, Q=$ $3, \tilde{\varepsilon}=4.9 \times 10^{-2}, \alpha=3.01, \beta=2.17$.

states of the system are the solutions of the cubic equation $-\phi\left\{1+\left[\delta_{0}+\left(1-\frac{\beta}{\alpha}\right) \phi\right]^{2}\right\}+\alpha=0$. By changing $\delta_{0}$, the system undergoes a Hopf bifurcation followed by a perioddoubling route to small-amplitude chaos to finally reach a chaotically spiking regime (see Fig. 4) as in the inertial vdPFN. The experimental verification of these results is currently in progress and will appear elsewhere.

In summary, we have shown that the addition of inertia to the fast dynamics of the vdPFN equations leads to a new scenario for the transition from excitability to relaxation oscillations. Here, a Hopf bifurcation followed by a perioddoubling cascade generate a multiplicity of periodic and chaotic excitable attractors that grow into a canard regime where they trigger erratic but deterministic sequences of spikes on top of a chaotic background. Our study opens a suggestive way to further generalize excitability by considering higher-dimensional fast manifolds where the fast dynamics itself could be chaotic, as well as complex combinations of relatively high dimensional slow and fast dynamics. We finally remark as an epistemological feature of this work that the study of a nonlinear optical system, critical in several advanced experiments, has motivated the development of a new mathematical body with results that return to physics as inspiration for new research. For instance, we expect that this new scenario will be relevant for biological applications, such as the study of some apparently erratic neural bursting that might have well defined coding functions.

This work was partially funded by the European Union ILIAS Project (No. RII3-CT-2003-506222), the CSICSpain Grant HIELOCRIS (No. 200530F0052). O. P. thanks Secretaria de Estado de Universidades e Investigacion del Ministerio de Educacion y Ciencias for additional financial help and Rockefeller University for hospitality.

[1] E. Izhikevich, Int. J. Bifurcation Chaos Appl. Sci. Eng. 10, 1171 (2000).

[2] J. M. Davidenko et al., Nature (London) 355, 349 (1992).

[3] A. N. Zaikin and A.M. Zhabotinskii, Nature (London) 225, 535 (1970).

[4] S. Barland, O. Piro, M. Giudici, J.R. Tredicce, and S. Balle, Phys. Rev. E 68, 036209 (2003).

[5] C. K. Jones and A.I. Khibnik, Multiple-Time-Scale Dynamic Systems, IMA Proceedings (Springer-Verlag, NY, 2000), Vol. 122.

[6] R. FitzHugh, Biophys. J. 1, 445 (1961); J. Nagumo, S. Arimoto, and S. Yoshizawa, Proc. IREE Aust. 50, 2061 (1962).

[7] M.W. Hirsch and S. Smale, Differential Equations, Dynamic Systems and Linear Algebra (Academic Press, NY, 1974).

[8] J. L. Callot, F. Diener, and M. Diener, C. R. Séances Acad. Sci. Ser. A, Math 286, 1059 (1978).

[9] V. A. Makarov, V. I. Nekorkin, and M. G. Velarde, Phys. Rev. Lett. 86, 3431 (2001).

[10] E. I. Volkov, E. Ullner, A. A. Zaikin, and J. Kurths, Phys. Rev. E 68, 026214 (2003).

[11] H. G. Rotstein, N. Kopell, A. M. Zhabotinskii, and I. R. Epstein, J. Chem. Phys. 119, 8824 (2003).

[12] F. Marino, G. Catalan, P. Sanchez, S. Balle, and O. Piro, Phys. Rev. Lett. 92, 073901 (2004).

[13] A.S. Pikovsky and M. I. Rabinovich, Physica D (Amsterdam) 2, 8 (1981).

[14] M.C. Eguia and G. B. Mindlin, Phys. Rev. E 60, 1551 (1999).

[15] R. Straube, D. Flockerzi, S. C. Muller, and M. J. B. Hauser, Phys. Rev. E 72, 066205 (2005)

[16] B. Deng, Chaos 14, 1083 (2004).

[17] S. Mancini, V. Giovannetti, D. Vitali, and P. Tombesi, Phys. Rev. Lett. 88, 120401 (2002).

[18] T. Corbitt et al., Phys. Rev. A 73, 023801 (2006).

[19] S. V. Dhurandhar, P. Hello, B. S. Sathyaprakash, and J. Y. Vinet, Appl. Opt. 36, 5325 (1997).

[20] C. Zhao, L. Ju, J. Degallaix, S. Gras, and D. G. Blair, Phys. Rev. Lett. 94, 121102 (2005).

[21] M. Feingold, D. L. Gonzalez, O. Piro, and H. Viturro, Phys. Rev. A 37, 4060 (1988).

[22] E. Yu Kalishin, M. M. Goncharenko, V. A. Khavrus, and P. E. Strizhak, Kinet. Catal. 43, 233 (2002).

[23] S. G. Lee, A. Neiman, and S. Kim, Phys. Rev. E 57, 3292 (1998).

[24] M. De Rosa, L. Conti, M. Cerdonio, M. Pinard, and F. Marin, Phys. Rev. Lett. 89, 237402 (2002).

[25] F. Marino, M. De Rosa, and F. Marin, Phys. Rev. E 73, 026217 (2006).

[26] With the replacements $Q^{2}=k, \tilde{\varepsilon} / Q=\varepsilon$, and the time scale change $\tau=Q^{2} t$, the right hand side of Eq. (5) reads $k \ddot{\phi}+\dot{\phi}$, as in Eq. (3). 